\title{
Permanence and global attractivity of a discrete pollination mutualism in plant-pollinator system with feedback controls
}

\author{
Rongyu Han', Xiangdong Xie ${ }^{2}$ and Fengde Chen²*
}

\section{"Correspondence:}

huanght0523@163.com

${ }^{2}$ Department of Mathematics,

Ningde Normal University, Ningde,

Fujian 352300, P.R. China

Full list of author information is

available at the end of the article

\section{Springer}

\begin{abstract}
In this paper, we propose a discrete pollination mutualism in a plant-pollinator system with the Beddington-DeAngelis functional response and feedback controls. By applying the comparison theorem of a difference equation and constructing some suitable Lyapunov functions, sufficient conditions are obtained for the permanence and the extinction of the system. Moreover, under some suitable conditions, we show that the solution of the system is globally attractive. The paper ends by some numerical simulations and a brief discussion.
\end{abstract}

MSC: 34C25; 92D25; 34D20; 34D40

Keywords: plant-pollinator system; feedback controls; permanence; extinction; global attractivity

\section{Introduction}

In theoretical ecology, there are several famous functional responses in the ecosystem, which we refer to as Holling type-I, type-II, type-III, type-IV, Monod-Haldane type, and Hassel-Verley type functional response etc. Some authors studied the ecosystem with different types of functional responses. Beddington [1] and DeAngelis et al. [2] first proposed a predator dependent functional response (known as the B-D functional response). After that, a lot of scholars did work on the ecosystems with the Beddington-DeAngelis functional response. Chen and You [3] studied the permanence, extinction, and periodic solution of the periodic predator-prey system with a Beddington-DeAngelis functional response and stage structure for prey. Xiao [4] analyzed the existence and uniqueness of the positive equilibrium and its global asymptotic stability by using the qualitative methods of ordinary differential equation. In [5], the author focused on the uniform persistence, local stability, and global stability for a Beddington-DeAngelis type stage structures predatorprey model. Furthermore, Chen et al. [6] with the help of a fluctuation lemma obtained a set of new conditions on the global asymptotic stability of the boundary solution.

In the ecological system, we know that it is more appropriate to use a discrete dynamic model to describe these systems when the populations have a short life or non-overlapping generations. Recently, Wu [7, 8] studied the permanence and global stability of a discrete

(c) 2016 Han et al. This article is distributed under the terms of the Creative Commons Attribution 4.0 International License (http://creativecommons.org/licenses/by/4.0/), which permits unrestricted use, distribution, and reproduction in any medium, provided you give appropriate credit to the original author(s) and the source, provide a link to the Creative Commons license, and indicate if changes were made. 
competition feedback-control system with a Beddington-DeAngelis functional response, moreover, she generalized it to $n$ species,

$$
\begin{aligned}
& x_{1}(t+1)=x_{1}(t) \exp \left\{r_{1}(t)-a_{1}(t) x_{1}(t)-\frac{c_{1}(t) x_{2}(t)}{1+x_{1}(t)+n(t) x_{2}(t)}-b_{1}(t) u_{1}(t)\right\}, \\
& x_{2}(t+1)=x_{2}(t) \exp \left\{r_{2}(t)-a_{2}(t) x_{2}(t)-\frac{c_{2}(t) x_{1}(t)}{1+x_{1}(t)+n(t) x_{2}(t)}-b_{2}(t) u_{2}(t)\right\}, \\
& \Delta u_{1}(t)=d_{1}(t)-p_{1}(t) u_{1}(t)+q_{1}(t) x_{1}(t), \\
& \Delta u_{2}(t)=d_{2}(t)-p_{2}(t) u_{2}(t)+q_{2}(t) x_{2}(t) .
\end{aligned}
$$

Li et al. [9] proposed a discrete predator-prey systems with a Beddington-DeAngelis functional response and feedback controls as follows:

$$
\begin{aligned}
& x(k+1)=x(k) \exp \left\{a(k)-b(k) x(k)-\frac{c(k) y(k)}{\alpha(k)+\beta(k) x(k)+\gamma(k) y(k)}-e_{1}(k) u_{1}(k)\right\}, \\
& y(k+1)=y(k) \exp \left\{-d(k)+\frac{f(k) x(k)}{\alpha(k)+\beta(k) x(k)+\gamma(k) y(k)}-e_{2}(k) u_{2}(k)\right\}, \\
& \Delta u_{1}(k)=-\eta_{1}(k) u_{1}(k)+q_{1}(k) x(k), \\
& \Delta u_{2}(k)=-\eta_{2}(k) u_{2}(k)+q_{2}(k) y(k) .
\end{aligned}
$$

By applying the comparison theorem of a difference equation, they obtained sufficient conditions to the permanence of the system.

Recently, Wang et al. [10] studied the interactions between pollinators, nectar robbers, defensive plants and non-defensive plants. Among them, the plant-pollinator system is described by a cooperative model with the Beddington-DeAngelis functional response,

$$
\begin{aligned}
& \frac{d x}{d t}=x\left(r_{1}-d_{1} x+\frac{\alpha_{12} y}{1+a x+b y}\right), \\
& \frac{d y}{d t}=y\left(-r_{2}+\frac{\alpha_{21} x}{1+a x+b y}\right),
\end{aligned}
$$

where $x$ and $y$ denote population densities of non-defensive plants and pollinators, respectively. $r_{1}$ represents the intrinsic growth rate of the plants and $r_{1} / d_{1}$ is their carrying capacity in the absence of visitors. $r_{2}$ denotes the pollinators' per capita mortality rate. Since the Beddington-DeAngelis functional response represents a positive effect of the pollinators on the plants, $\alpha_{12}$ can be regarded as the plants' efficiency in translating the plant-pollinator interactions into fitness. Similarly, $\alpha_{21}$ represents the corresponding value for the pollinators. The authors analyzed the global stability of the positive equilibrium of the system.

On the other hand, as was pointed out by Huo and Li [11], ecosystems in the real world are continuously disrupted by unpredictable forces which can result in changes in the biological parameters. For having a more accurate description of such a system, scholars introduced the feedback control into the ecosystems. Moreover, discrete time models, governed by difference equations, are more appropriate than the continuous ones. The above phenomena motivated us to propose and study the discrete non-autonomous pollination mutualism in a plant-pollinator system with a Beddington-DeAngelis functional 
response and feedback controls as follows:

$$
\begin{aligned}
& x_{1}(n+1)=x_{1}(n) \exp \left\{r_{1}(n)-d_{1}(n) x_{1}(n)+\frac{\alpha_{12}(n) x_{2}(n)}{1+a(n) x_{1}(n)+b(n) x_{2}(n)}-e_{1}(n) u_{1}(n)\right\}, \\
& x_{2}(n+1)=x_{2}(n) \exp \left\{-r_{2}(n)+\frac{\alpha_{21}(n) x_{1}(n)}{1+a(n) x_{1}(n)+b(n) x_{2}(n)}-e_{2}(n) u_{2}(n)\right\}, \\
& \Delta u_{1}(n)=-\eta_{1}(n) u_{1}(n)+q_{1}(n) x_{1}(n), \\
& \Delta u_{2}(n)=-\eta_{2}(n) u_{2}(n)+q_{2}(n) x_{2}(n),
\end{aligned}
$$

where $x_{i}(n)(i=1,2)$ is the density of $x_{i}$ species at the $n$th generation and $u_{i}(n)(i=1,2)$ is the feedback-control variable. The coefficients $r_{i}(n), d_{1}(n), a(n), b(n), \alpha_{i j}(n), e_{i}(n), \eta_{i}(n)$, $q_{i}(n)(i, j=1,2, i \neq j)$ are all bounded nonnegative sequences.

Throughout this paper, we use the following notations for any bounded sequence $\{f(n)\}$ :

$$
f^{u}=\sup _{n \in N} f(n), \quad f^{l}=\inf _{n \in N} f(n),
$$

and assume that $0<\eta_{i}^{l} \leq \eta_{i}^{u}<1(i=1,2)$.

According to the biological background of system (1.4), we only consider the solution of the system (1.4) with the following initial conditions:

$$
x_{i}(0)>0 ; \quad u_{i}(0)>0, \quad i=1,2 .
$$

It is easy to prove that the solution of the system (1.4) which satisfies the initial condition is positive.

We mention here that, as far as the system (1.4) is concerned, whether the system is to persist is a question that we need to solve. Furthermore whether the feedback-control variables have influence on the extinction and the stability of the system or not is an interesting problem. The aim of this paper is to solve the above questions.

The remainder of the paper is organized as follows: in Section 2, we introduce some useful lemmas and obtain the sufficient conditions to guarantee the permanence and the extinction of system (1.4). In Section 3, a set of sufficient conditions which ensure the stability of the system are obtained. In Section 4, we give some examples to illustrate our results, and we end this paper by a brief discussion.

\section{Permanence}

In this section, we establish a permanence result for system (1.4). First, let us state several lemmas which will be useful in proving the main results.

Now let us consider the first order difference equation

$$
y(n+1)=A y(n)+B, \quad n=1,2, \ldots,
$$

where $A$ and $B$ are positive constants.

Lemma 2.1 [12] Assume that $|A|<1$, for any initial value $y(0)$, there exists a unique solution $y(n)$ of (2.1) which can be expressed as follows:

$$
y(n)=A^{n}\left(y(0)-y^{*}\right)+y^{*},
$$


where $y^{*}=B /(1-A)$. Thus, for any solution $y(n)$ of system $(2.1)$,

$$
\lim _{n \rightarrow+\infty} y(n)=y^{*}
$$

Lemma 2.2 [12] Let $n \in N_{n_{0}}^{+}=\left\{n_{0}, n_{0}+1, \ldots, n_{0}+l, \ldots\right\}, r \geq 0$. For any fixed $n, g(n, r)$ is a nondecreasing function with respect to $r$, and for $n \geq n_{0}$, the following inequalities hold:

$$
y(n+1) \leq g(n, y(n)), \quad u(n+1) \geq g(n, u(n)) .
$$

If $y\left(n_{0}\right) \leq u\left(n_{0}\right)$, then $y(n) \leq u(n)$ for all $n \geq n_{0}$.

Now let us consider the following single species discrete model:

$$
N(n+1)=N(n) \exp (a(n)-b(n) N(n))
$$

where $a(n)$ and $b(n)$ are strictly positive sequences of real numbers defined for $n \in N=$ $\{0,1,2, \ldots\}$ and $0<a^{l} \leq a^{u}, 0<b^{l} \leq b^{u}$. We have the following lemma.

Lemma 2.3 Any solution of system (2.5) with initial condition $N(0)>0$ satisfies

$$
m \leq \liminf _{n \rightarrow+\infty} N(n) \leq \limsup _{n \rightarrow+\infty} N(n) \leq M,
$$

where

$$
M=\frac{1}{b^{l}} \exp \left(a^{u}-1\right), \quad m=\frac{a^{l}}{b^{u}} \exp \left(a^{l}-b^{u} M\right) .
$$

Lemma 2.4 [13] Let $x(n)$ and $b(n)$ be nonnegative sequences defined on $N$ and $c \geq 0$ is $a$ constant. If

$$
x(n) \leq c+\sum_{s=0}^{n-1} b(s) x(s), \quad \text { for } n \in N
$$

then

$$
x(n) \leq c \prod_{s=0}^{n-1}[1+b(s)], \quad \text { for } n \in N
$$

Lemma 2.5 [14] Assume that $A>0$ and $y(0)>0$, and further suppose that

$$
y(n+1) \leq A y(n)+B(n), \quad n=1,2, \ldots
$$

Then, for any integer $k \leq n$,

$$
y(n) \leq A^{k} y(n-k)+\sum_{i=0}^{k-1} A^{i} B(n-i-1) .
$$


Proposition 2.6 Assume that

$$
-r_{2}^{l}+\frac{\alpha_{21}^{u}}{a^{l}}>0
$$

holds, then

$$
\limsup _{n \rightarrow+\infty} x_{i}(n) \leq M_{i}, \quad \limsup _{n \rightarrow+\infty} u_{i}(n) \leq W_{i}, \quad i=1,2,
$$

where

$$
\begin{aligned}
& M_{1}=\frac{1}{d_{1}^{l}} \exp \left\{r_{1}^{u}+\frac{\alpha_{12}^{u}}{b^{l}}-1\right\}, \\
& M_{2}=\exp \left\{2\left(-r_{2}^{l}+\frac{\alpha_{21}^{u}}{a^{l}}\right)\right\}, \\
& W_{i}=\frac{r_{i}^{u} M_{i}}{\eta_{i}^{l}} \quad(i=1,2) .
\end{aligned}
$$

Proof Let $\left(x_{1}(n), x_{2}(n), u_{1}(n), u_{2}(n)\right)$ be any positive solution of system (1.4), it follows from the first equation of system (1.4) that

$$
x_{1}(n+1) \leq x_{1}(n) \exp \left\{r_{1}(n)-d_{1}(n) x_{1}(n)+\frac{\alpha_{12}(n)}{b(n)}\right\} .
$$

By applying Lemmas 2.2 and 2.3, we have

$$
\limsup _{n \rightarrow+\infty} x_{1}(n) \leq \frac{1}{d_{1}^{l}} \exp \left\{r_{1}^{u}+\frac{\alpha_{12}^{u}}{b^{l}}-1\right\} \stackrel{\text { def }}{=} M_{1} .
$$

Let $x_{2}(n)=\exp \{v(n)\}$, then

$$
\begin{aligned}
v(n+1) & \leq v(n)+\left(-r_{2}^{l}+\frac{\alpha_{21}^{u}}{a^{l}}\right) \\
& =\sum_{s=0}^{n} c(s) v(s)+\left(-r_{2}^{l}+\frac{\alpha_{21}^{u}}{a^{l}}\right),
\end{aligned}
$$

where

$$
c(s)= \begin{cases}0, & 0 \leq s \leq n-1, \\ 1, & s=n .\end{cases}
$$

Condition (2.10) shows that Lemma 2.4 could be applied to (2.12), it immediately follows that

$$
v(n+1) \leq 2\left(-r_{2}^{l}+\frac{\alpha_{21}^{u}}{a^{l}}\right) .
$$

This is

$$
\limsup _{n \rightarrow+\infty} x_{2}(n) \leq \exp \left\{2\left(-r_{2}^{l}+\frac{\alpha_{21}^{u}}{a^{l}}\right)\right\} \stackrel{\text { def }}{=} M_{2} .
$$


For any small enough positive constant $\varepsilon$, it follows from (2.11) and (2.13) that there exists a large enough $N_{0}$ such that

$$
x_{i}(n) \leq M_{i \varepsilon}, \quad \forall n \geq N_{0}, i=1,2 .
$$

From the third and fourth equations of the system (1.4) and (2.14), we can obtain

$$
\begin{aligned}
& u_{1}(n+1) \leq\left(1-\eta_{1}^{l}\right) u_{1}(n)+q_{1}^{u} M_{1 \varepsilon}, \\
& u_{2}(n+1) \leq\left(1-\eta_{2}^{l}\right) u_{1}(n)+q_{2}^{u} M_{2 \varepsilon} .
\end{aligned}
$$

By applying Lemmas 2.1 and 2.2 , it immediately follows that

$$
\begin{aligned}
& \limsup _{n \rightarrow+\infty} u_{1}(n) \leq \frac{q_{1}^{u} M_{1 \varepsilon}}{\eta_{1}^{l}}, \\
& \limsup _{n \rightarrow+\infty} u_{2}(n) \leq \frac{q_{2}^{u} M_{2 \varepsilon}}{\eta_{2}^{l}} .
\end{aligned}
$$

Setting $\varepsilon \rightarrow 0$ in the above inequalities leads to

$$
\begin{aligned}
& \limsup _{n \rightarrow+\infty} u_{1}(n) \leq \frac{q_{1}^{u} M_{1}}{\eta_{1}^{l}} \stackrel{\text { def }}{=} W_{1}, \\
& \limsup _{n \rightarrow+\infty} u_{2}(n) \leq \frac{q_{2}^{u} M_{2}}{\eta_{2}^{l}} \stackrel{\text { def }}{=} W_{2} .
\end{aligned}
$$

This completes the proof of Proposition 2.6.

Theorem 2.7 In addition to (2.10), assume further that

$$
-r_{2}^{u}+\frac{\alpha_{21}^{l} m_{1}}{1+a^{u} m_{1}}-e_{1}^{u} W_{2}>0,
$$

then the system (1.4) is permanent.

Proof By applying Proposition 2.6, it is easy to see that, to end the proof of Theorem 2.7, it is enough to show that under the conditions of Theorem 2.7,

$$
\liminf _{n \rightarrow+\infty} x_{i}(n) \geq m_{i}, \quad \liminf _{n \rightarrow+\infty} u_{i}(n) \geq w_{i}, \quad i=1,2 .
$$

From Proposition 2.6, we know that for the above $\varepsilon$, there exists a $N_{1}>N_{0}$ such that

$$
x_{i}(n) \leq M_{i \varepsilon}, \quad u_{i}(n) \leq W_{i \varepsilon}, \quad i=1,2 \text { for all } n>N_{1} .
$$

From the first equation of system (1.4) and (2.16), we have

$$
\begin{aligned}
x_{1}(n+1) & \geq x_{1}(n) \exp \left\{r_{1}^{l}-d_{1}^{u} M_{1 \varepsilon}-e_{1}^{u} W_{1 \varepsilon}\right\} \\
& \geq x_{1}(n) \exp \left\{-d_{1}^{u} M_{1 \varepsilon}-e_{1}^{u} W_{1 \varepsilon}\right\} \\
& \stackrel{\text { def }}{=} x_{1}(n) \exp \left\{D_{\varepsilon}\right\}
\end{aligned}
$$


for all $n>N_{1}$, where $D_{\varepsilon}=-d_{1}^{u} M_{1 \varepsilon}-e_{1}^{u} W_{1 \varepsilon}$. So for $n \geq k$ we have

$$
x_{1}(n-k) \leq x_{1}(n) \exp \left\{-D_{\varepsilon} k\right\} .
$$

From the third equation of system (1.4), we have

$$
\begin{aligned}
u_{1}(n+1) & \leq\left(1-\eta_{1}^{l}\right) u_{1}(n)+q_{1}^{u} x_{1}(n) \\
& \stackrel{\text { def }}{=} A u_{1}(n)+B(n)
\end{aligned}
$$

where $A=1-\eta_{1}^{l}, B=q_{1}^{u} x_{1}(n)$. Then Lemma 2.5 implies that, for any $n \geq k$,

$$
\begin{aligned}
u_{1}(n) & \leq A^{k} u_{1}(n-k)+\sum_{i=0}^{k-1} A^{i} B(n-i-1) \\
& =A^{k} u_{1}(n-k)+\sum_{i=0}^{k-1} A^{i} q_{1}^{u} x_{1}(n-i-1) \\
& \leq A^{k} u_{1}(n-k)+q_{1}^{u} x_{1}(n) \sum_{i=0}^{k-1} A^{i} \exp \left\{-D_{\varepsilon}(i+1)\right\} .
\end{aligned}
$$

Note that

$$
0 \leq A^{k} u_{1}(n-k) \leq A^{k} W_{1 \varepsilon} \rightarrow 0, \quad k \rightarrow+\infty
$$

We can choose $N_{2}=\max \left\{N_{1}, \frac{\ln P_{1}}{\ln A}\right\}+1$, where $P_{1}=\frac{r_{1}^{u}}{e_{1}^{u} W_{1 \varepsilon}}$. As $n>N_{2}$, we have $r_{1}^{l}-e_{1}^{u} A^{N_{2}} W_{1 \varepsilon}>$ 0 , then we get

$$
\begin{aligned}
u_{1}(n) & \leq A^{N_{2}} u_{1}\left(n-N_{2}\right)+q_{1}^{u} x_{1}(n) \sum_{i=0}^{N_{2}-1} A^{i} \exp \left\{-D_{\varepsilon}(i+1)\right\} \\
& \leq A^{N_{2}} W_{1 \varepsilon}+q_{1}^{u} x_{1}(n) \sum_{i=0}^{N_{2}-1} A^{i} \exp \left\{-D_{\varepsilon}(i+1)\right\} \\
& \stackrel{\text { def }}{=} A^{N_{2}} W_{1 \varepsilon}+G_{\varepsilon} x_{1}(n),
\end{aligned}
$$

where $G_{\varepsilon}=q_{1}^{u} \sum_{i=0}^{N_{2}-1} A^{i} \exp \left\{-D_{\varepsilon}(i+1)\right\}$.

Considering the first equation of system (1.4), we have

$$
\begin{aligned}
x_{1}(n+1) & \geq x_{1}(n) \exp \left\{r_{1}^{l}-d_{1}^{u} x_{1}(n)-e_{1}^{u} u_{1}(n)\right\} \\
& \geq x_{1}(n) \exp \left\{r_{1}^{l}-e_{1}^{u}\left(A^{N_{2}} W_{1 \varepsilon}+G_{\varepsilon} x_{1}(n)\right)-d_{1}^{u} x_{1}(n)\right\} \\
& =x_{1}(n) \exp \left\{r_{1}^{l}-e_{1}^{u} A^{N_{2}} W_{1 \varepsilon}-\left(e_{1}^{u} G_{\varepsilon}+d_{1}^{u}\right) x_{1}(n)\right\} \\
& \stackrel{\operatorname{def}}{=} x_{1}(n) \exp \left\{E_{1 \varepsilon}-E_{2 \varepsilon} x_{1}(n)\right\},
\end{aligned}
$$

where $E_{1 \varepsilon}=r_{1}^{l}-e_{1}^{u} A^{N_{2}} W_{1 \varepsilon}, E_{2 \varepsilon}=e_{1}^{u} G_{\varepsilon}+d_{1}^{u}$. 
By applying Lemmas 2.2 and 2.3, it immediately follows that

$$
\liminf _{n \rightarrow+\infty} x_{1}(n) \geq \frac{E_{1 \varepsilon}}{E_{2 \varepsilon}} \exp \left\{E_{1 \varepsilon}-E_{2 \varepsilon} M_{1}\right\} .
$$

Setting $\varepsilon \rightarrow 0$ in (2.17) leads to

$$
\liminf _{n \rightarrow+\infty} x_{1}(n) \geq \frac{E_{1}}{E_{2}} \exp \left\{E_{1}-E_{2} M_{1}\right\} \stackrel{\text { def }}{=} m_{1} .
$$

Then we assume that $\varepsilon<\frac{1}{2} m_{1}$, from (2.18) we know that there exists a large enough $N_{2}>N_{1}$ such that

$$
x_{1}(n) \geq m_{1 \varepsilon}, \quad \forall n \geq T_{2}
$$

From the second equation of system (1.4), (2.16), and (2.19), we have

$$
\begin{aligned}
x_{2}(n+1)= & x_{2}(n) \exp \left\{-r_{2}(n)+\frac{\alpha_{21}(n)}{a(n)}-\frac{\alpha_{21}(n)}{a(n)}\right. \\
& \left.\times\left(\frac{1+b(n) x_{2}(n)}{1+a(n) x_{1}(n)+b(n) x_{2}(n)}\right)-e_{2}(n) u_{2}(n)\right\} \\
\geq & x_{2}(n) \exp \left\{-r_{2}(n)+\frac{\alpha_{21}(n)}{a(n)}-\frac{\alpha_{21}(n)}{a(n)\left(1+a(n) m_{1 \varepsilon}\right)}\right. \\
& \left.-e_{2}(n) W_{2 \varepsilon}-\frac{\alpha_{21}(n) b(n)}{a(n)\left(1+a(n) m_{1 \varepsilon}\right)} x_{2}(n)\right\} \\
= & x_{2}(n) \exp \left\{-r_{2}(n)+\frac{\alpha_{21}(n) m_{1 \varepsilon}}{1+a(n) m_{1 \varepsilon}}-e_{2}(n) W_{2 \varepsilon}\right. \\
& \left.-\frac{\alpha_{21}(n) b(n)}{a(n)\left(1+a(n) m_{1 \varepsilon}\right)} x_{2}(n)\right\}
\end{aligned}
$$

for all $n>N_{2}$.

By applying Lemmas 2.2 and 2.3, it immediately follows that

$$
\begin{aligned}
\liminf _{n \rightarrow+\infty} x_{2}(n) \geq & \frac{\left[\left(\frac{\alpha_{21}^{l} m_{1 \varepsilon}}{1+a^{u} m_{1 \varepsilon}}\right)-r_{2}^{u}-e_{2}^{u} W_{2 \varepsilon}\right]\left(a^{l}\left(1+a^{l} m_{1 \varepsilon}\right)\right)}{\alpha_{21}^{u} b^{u}} \\
& \times \exp \left\{\frac{\alpha_{21}^{l} m_{1 \varepsilon}}{1+a^{u} m_{1 \varepsilon}}-r_{2}^{u}-e_{2}^{u} W_{2 \varepsilon}-\frac{\alpha_{21}^{u} b^{u}}{a^{l}\left(1+a^{l} m_{1 \varepsilon}\right)} M_{2}\right\} .
\end{aligned}
$$

Setting $\varepsilon \rightarrow 0$, we have

$$
\begin{aligned}
\liminf _{n \rightarrow+\infty} x_{2}(n) \geq & \frac{\left[\left(\frac{\alpha_{21}^{l} m_{1}}{1+a^{u} m_{1}}\right)-r_{2}^{u}-e_{2}^{u} W_{2}\right]\left(a^{l}\left(1+a^{l} m_{1}\right)\right)}{\alpha_{21}^{u} b^{u}} \\
& \times \exp \left\{\frac{\alpha_{21}^{l} m_{1}}{1+a^{u} m_{1}}-r_{2}^{u}-e_{2}^{u} W_{2}-\frac{\alpha_{21}^{u} b^{u}}{a^{l}\left(1+a^{l} m_{1}\right)} M_{2}\right\} \stackrel{\text { def }}{=} m_{2} .
\end{aligned}
$$

Without loss of generality, we may assume that $\varepsilon<(1 / 2) \min \left\{m_{1}, m_{2}\right\}$. It follows from (2.18) and (2.21) that there exists a large enough $N_{3}>N_{2}$, such that

$$
x_{i} \geq m_{i \varepsilon}, \quad i=1,2, \forall n \geq N_{3} .
$$


From the third and fourth equations of the system (1.4) and (2.22), we have

$$
\begin{aligned}
& u_{1}(n+1) \geq\left(1-\eta_{1}^{u}\right) u_{1}(n)+q_{1}^{l} m_{1 \varepsilon}, \\
& u_{2}(n+1) \geq\left(1-\eta_{2}^{u}\right) u_{1}(n)+q_{2}^{l} m_{2 \varepsilon} .
\end{aligned}
$$

By applying Lemmas 2.1 and 2.2 , it immediately follows that

$$
\begin{aligned}
& \liminf _{n \rightarrow+\infty} u_{1}(n) \geq \frac{q_{1}^{l} m_{1 \varepsilon}}{\eta_{1}^{u}}, \\
& \liminf _{n \rightarrow+\infty} u_{2}(n) \geq \frac{q_{2}^{l} m_{2 \varepsilon}}{\eta_{2}^{u}} .
\end{aligned}
$$

Setting $\varepsilon \rightarrow 0$ in the above inequalities leads to

$$
\begin{aligned}
& \liminf _{n \rightarrow+\infty} u_{1}(n) \geq \frac{q_{1}^{l} m_{1}}{\eta_{1}^{u}} \stackrel{\text { def }}{=} w_{1}, \\
& \liminf _{n \rightarrow+\infty} u_{2}(n) \geq \frac{q_{2}^{l} m_{2}}{\eta_{2}^{u}} \stackrel{\text { def }}{=} w_{2} .
\end{aligned}
$$

This completes the proof.

Theorem 2.8 Assume that the inequality

$$
r_{2}^{l}>\frac{\alpha_{21}^{u}}{a^{l}}
$$

holds. Let $\left(x_{1}(n), x_{2}(n), u_{1}(n), u_{2}(n)\right)$ be any positive solution of system $(1.4)$, then $x_{2}(n) \rightarrow 0$, $u_{2}(n) \rightarrow 0$ as $n \rightarrow+\infty$.

Proof Equation (2.23) is equivalent to the following inequality:

$$
-r_{2}^{l}+\frac{\alpha_{21}^{u}}{a^{l}}<0
$$

From (2.24), there exists a $\delta>0$ such that

$$
-r_{2}^{l}+\frac{\alpha_{21}^{u}}{a^{l}}<-\delta<0
$$

Let $\left(x_{1}(n), x_{2}(n), u_{1}(n), u_{2}(n)\right)$ be any positive solution of system (1.4). For any $q \in N$, according to the second equation of system (1.4), we obtain

$$
\begin{aligned}
\ln \frac{x_{2}(q+1)}{x_{2}(q)} & =-r_{2}(q)+\frac{\alpha_{21}(q) x_{1}(q)}{1+a(q) x_{1}(q)+b(q) x_{2}(q)}-e_{2}(q) u_{2}(q) \\
& \leq r_{2}(q)+\frac{\alpha_{21}(q) x_{1}(q)}{1+a(q) x_{1}(q)+b(q) x_{2}(q)} \\
& \leq-r_{2}^{l}+\frac{\alpha_{21}^{u}}{a^{l}}<-\delta<0 .
\end{aligned}
$$


Summating both sides of the above inequalities from 0 to $n-1$, we obtain

$$
\ln \frac{x_{2}(n)}{x_{2}(0)}<-\delta n
$$

then

$$
x_{2}(n)<x_{2}(0) \exp \{-\delta n\} .
$$

From (2.27), $x_{2}(n) \rightarrow 0$ as $n \rightarrow+\infty$.

Further, consider the fourth equation of system (1.4). Applying Lemma 2.3 in [15], we easily obtain $u_{2}(n) \rightarrow 0$ as $n \rightarrow+\infty$. This completes the proof of Theorem 2.8.

Theorem 2.9 Assume that (2.23) holds, and further assume that

$$
\begin{aligned}
& \eta_{1}^{l}>e_{1}^{u}, \\
& \min \left[d_{1}^{l}, \frac{2}{M_{1}}-d_{1}^{u}\right]>q_{1}^{u},
\end{aligned}
$$

then, for any two positive solutions $\left(x_{1}(n), x_{2}(n), u_{1}(n), u_{2}(n)\right)$ and $\left(x_{1}^{*}(n), x_{2}^{*}(n), u_{1}^{*}(n), u_{2}^{*}(n)\right)$ of the system, we have

$$
\lim _{n \rightarrow+\infty}\left(x_{1}(n)-x_{1}^{*}(n)\right)=0, \quad \lim _{n \rightarrow+\infty}\left(u_{1}(n)-u_{1}^{*}(n)\right)=0 .
$$

Proof By conditions (2.28), there exist a positive constant $\varepsilon$ and $\delta$ such that

$$
\begin{aligned}
& \eta_{1}^{l}-e_{1}^{u}>\delta \\
& \min \left[d_{1}^{l}, \frac{2}{M_{1 \varepsilon}}-d_{1}^{u}\right]-q_{1}^{u}>\delta .
\end{aligned}
$$

From Theorems 2.7 and 2.8, for the above $\varepsilon$, there exists $N_{4}>N_{3}$ such that

$$
m_{i \varepsilon} \leq x_{1}(n), \quad x_{1}^{*}(n) \leq M_{i \varepsilon}, \quad x_{2}(n) \leq \varepsilon .
$$

Using the mean value theorem, one has

$$
\ln x_{1}(n)-\ln x_{1}^{*}(n)=\frac{1}{\theta(n)}\left(x_{1}(n)-x_{1}^{*}(n)\right),
$$

where $\theta(n)$ is between $x_{1}(n)$ and $x_{1}^{*}(n)$.

Now we define

$$
V_{1}(n)=\left|\ln x_{1}(n)-\ln x_{1}^{*}(n)\right|, \quad V_{2}(n)=\left|u_{1}(n)-u_{1}^{*}(n)\right| .
$$

From the first equation of the system, we have

$$
\begin{aligned}
\Delta V_{1}(n) & =\left|\ln x_{1}(n+1)-\ln x_{1}^{*}(n+1)\right|-\left|\ln x_{1}(n)-\ln x_{1}^{*}(n)\right| \\
& \leq\left|\ln x_{1}(n)-\ln x_{1}^{*}(n)-d_{1}(n)\left(x_{1}(n)-x_{1}^{*}(n)\right)\right|
\end{aligned}
$$




$$
\begin{aligned}
& -\left|\ln x_{1}(n)-\ln x_{1}^{*}(n)\right|+\alpha_{12}(n) \mid \frac{x_{2}(n)}{1+a(n) x_{1}(n)+b(n) x_{2}(n)} \\
& -\frac{x_{2}^{*}(n)}{1+a(n) x_{1}^{*}(n)+b(n) x_{2}^{*}(n)}\left|+e_{1}(n)\right| u_{1}(n)-u_{1}^{*}(n) \mid \\
\leq & -\left(\frac{1}{\theta(n)}-\left|\frac{1}{\theta(n)}-d_{1}(n)\right|\right)\left|x_{1}(n)-x_{1}^{*}(n)\right| \\
& +e_{1}^{u}\left|u_{1}(n)-u_{1}^{*}(n)\right|+\alpha_{12}^{u} \frac{x_{2}(n)+x_{2}^{*}(n)}{1+a^{l} m_{1 \varepsilon}+b^{l} \varepsilon}
\end{aligned}
$$

From the third equation of the system, we have

$$
\begin{aligned}
\Delta V_{2}(n) & =\left|u_{1}(n+1)-u_{1}^{*}(n+1)\right|-\left|u_{1}(n)-u_{1}^{*}(n)\right| \\
& \leq-\eta_{1}^{l}\left|u_{1}(n)-u_{1}^{*}(n)\right|+q_{1}^{u}\left|x_{1}(n)-x_{1}^{*}(n)\right| .
\end{aligned}
$$

Now we define a Lyapunov function as follows:

$$
V(n)=V_{1}(n)+V_{2}(n)
$$

From (2.31) and (2.32), we have

$$
\begin{aligned}
\Delta V(n) \leq & -\left\{\min \left[d_{1}^{l}, \frac{2}{M_{1 \varepsilon}}-d_{1}^{u}\right]-q_{1}^{u}\right\}\left|x_{1}(n)-x_{1}^{*}(n)\right|-\left\{\eta_{1}^{l}-e_{1}^{u}\right\} \\
& \times\left|u_{1}(n)-u_{1}^{*}(n)\right|+\alpha_{12}^{u} \frac{x_{2}(n)+x_{2}^{*}(n)}{1+a^{l} m_{1 \varepsilon}+b^{l} \varepsilon} \\
\leq & -\delta\left(\left|x_{1}(n)-x_{1}^{*}(n)\right|+\left|u_{1}(n)-u_{1}^{*}(n)\right|\right)+\alpha_{12}^{u} \frac{x_{2}(n)+x_{2}^{*}(n)}{1+a^{l} m_{1 \varepsilon}+b^{l} \varepsilon} .
\end{aligned}
$$

Summating both sides of the above inequalities from $N_{4}$ to $n$, we have

$$
\begin{aligned}
\sum_{p=N_{4}}^{n}(V(p+1)-V(p)) \leq & -\delta \sum_{p=N_{4}}^{n}\left(\left|x_{1}(n)-x_{1}^{*}(n)\right|+\left|u_{1}(n)-u_{1}^{*}(n)\right|\right) \\
& +\frac{\alpha_{12}^{u}}{1+a^{l} m_{1 \varepsilon}+b^{l} \varepsilon} \sum_{p=N_{4}}^{n}\left(x_{2}(n)+x_{2}^{*}(n)\right) .
\end{aligned}
$$

Hence

$$
\begin{gathered}
V(n+1)+\delta \sum_{p=N_{4}}^{n}\left(\left|x_{1}(n)-x_{1}^{*}(n)\right|+\left|u_{1}(n)-u_{1}^{*}(n)\right|\right) \\
\leq V\left(N_{4}\right)+\frac{\alpha_{12}^{u}}{1+a^{l} m_{1 \varepsilon}+b^{l} \varepsilon} \sum_{p=N_{4}}^{n}\left(x_{2}(n)+x_{2}^{*}(n)\right) .
\end{gathered}
$$

From Theorem 2.8, we have $\sum_{n=0}^{+\infty} x_{2}(n)<+\infty$ and $\sum_{n=0}^{+\infty} x_{2}^{*}(n)<+\infty$. We notice that $V\left(N_{4}\right)$ is bounded. So from the above inequalities, we have

$$
\sum_{p=N_{4}}^{n}\left(\left|x_{1}(n)-x_{1}^{*}(n)\right|+\left|u_{1}(n)-u_{1}^{*}(n)\right|\right) \leq+\infty .
$$


Therefore

$$
\sum_{p=N_{4}}^{+\infty}\left(\left|x_{1}(n)-x_{1}^{*}(n)\right|+\left|u_{1}(n)-u_{1}^{*}(n)\right|\right) \leq+\infty .
$$

This means that

$$
\lim _{n \rightarrow+\infty}\left(\left|x_{1}(n)-x_{1}^{*}(n)\right|+\left|u_{1}(n)-u_{1}^{*}(n)\right|\right)=0 .
$$

Consequently

$$
\begin{aligned}
& \lim _{n \rightarrow+\infty}\left(x_{1}(n)-x_{1}^{*}(n)\right)=0, \\
& \lim _{n \rightarrow+\infty}\left(u_{1}(n)-u_{1}^{*}(n)\right)=0 .
\end{aligned}
$$

This completes the proof of Theorem 2.9.

\section{Global attractivity}

In this section, we will consider the stability of the system (1.4).

Theorem 3.1 In addition to the conditions of Theorem 2.7, assume that the following condition holds:

$$
\left\{\begin{aligned}
\chi_{1}= & \max \left\{\left|1-d_{1}^{l} m_{1}-\frac{a^{l} \alpha_{12}^{l} m_{1} m_{2}}{\Delta\left(n, M_{1}, m_{2}\right)}\right|,\left|1-d_{1}^{u} M_{1}-\frac{a^{u} \alpha_{12}^{u} M_{1} M_{2}}{\Delta\left(n, m_{1}, M_{2}\right)}\right|\right\} \\
& +\alpha_{12}^{u}\left[\frac{M_{2}}{\Delta\left(n, m_{1}, m_{2}\right)}+\frac{a^{u} M_{1} M_{2}}{\Delta\left(n, M_{1}, m_{2}\right)}\right]+e_{1}^{u}<1 \\
\chi_{2}= & \max \left\{\left|1-\frac{b^{l} \alpha_{21}^{l} m_{1} m_{2}}{\Delta\left(n, m_{1}, M_{2}\right)}\right|,\left|1-\frac{b^{u} \alpha_{21}^{u} M_{1} M_{2}}{\Delta\left(n, M_{1}, m_{2}\right)}\right|\right\} \\
& +\alpha_{21}^{u}\left[\frac{M_{1}}{\Delta\left(n, m_{1}, m_{2}\right)}+\frac{b^{u} M_{1} M_{2}}{\Delta\left(n, m_{1}, M_{2}\right)}\right]+e_{2}^{u}<1 \\
\chi_{3}= & 1-\eta_{1}^{l}+q_{1}^{u} M_{1}<1 \\
\chi_{4}= & 1-\eta_{2}^{l}+q_{2}^{u} M_{2}<1
\end{aligned}\right.
$$

where $m_{i}, M_{i}, i=1,2$, are defined as before and

$$
\Delta\left(n, x_{1}(n), x_{2}(n)\right)=\left[1+a(n) x_{1}(n)+b(n) x_{2}(n)\right]\left[1+a(n) x_{1}^{*}(n)+b(n) x_{2}^{*}(n)\right],
$$

then the solution $\left(x_{1}(n), x_{2}(n), u_{1}(n), u_{2}(n)\right)$ of the system (1.4) is globally attractive.

Proof Let $\left(x_{1}(n), x_{2}(n), u_{1}(n), u_{2}(n)\right)$ and $\left(x_{1}^{*}(n), x_{2}^{*}(n), u_{1}^{*}(n), u_{2}^{*}(n)\right)$ be any two positive solutions of the system (1.4), let

$$
W_{i}(n)=\left|\ln x_{i}(n)-\ln x_{i}^{*}(n)\right|, \quad V_{i}(n)=\left|u_{i}(n)-u_{i}^{*}(n)\right|, \quad i=1,2 .
$$

So from the first equation of the system, we have

$$
\begin{aligned}
W_{1}(n+1)= & \operatorname{sgn}\left(x_{1}(n)-x_{1}^{*}(n)\right)\left\{\ln x_{1}(n)+r_{1}(n)-d_{1}(n) x_{1}(n)\right. \\
& +\frac{\alpha_{12}(n) x_{2}(n)}{1+a(n) x_{1}(n)+b(n) x_{2}(n)}-e_{1}(n) u_{1}(n)-\ln x_{1}^{*}(n)
\end{aligned}
$$




$$
\begin{aligned}
& \left.-\left[r_{1}(n)-d_{1}(n) x_{1}^{*}(n)+\frac{\alpha_{12}(n) x_{2}^{*}(n)}{1+a(n) x_{1}^{*}(n)+b(n) x_{2}^{*}(n)}-e_{1}(n) u_{1}^{*}(n)\right]\right\} \\
\leq & \left|\left(\ln x_{1}(n)-\ln x_{1}^{*}(n)\right)-d_{1}(n)\left(x_{1}(n)-x_{1}^{*}(n)\right)\right|+e_{1}^{u} V_{1}(n) \\
& +\alpha_{12}^{u} \operatorname{sgn}\left(x_{1}(n)-x_{1}^{*}(n)\right) \times\left\{\frac{x_{2}(n)}{1+a(n) x_{1}(n)+b(n) x_{2}(n)}\right. \\
& \left.-\frac{x_{2}^{*}(n)}{1+a(n) x_{1}^{*}(n)+b(n) x_{2}^{*}(n)}\right\} .
\end{aligned}
$$

In a similar way, we get

$$
\begin{aligned}
W_{2}(n+1) \leq & \left|\ln x_{2}(n)-\ln x_{2}^{*}(n)\right|+e_{2}^{u} V_{2}(n)+\alpha_{21}^{u} \operatorname{sgn}\left(x_{2}(n)-x_{2}^{*}(n)\right) \\
& \times\left\{\frac{x_{1}(n)}{1+a(n) x_{1}(n)+b(n) x_{2}(n)}-\frac{x_{1}^{*}(n)}{1+a(n) x_{1}^{*}(n)+b(n) x_{2}^{*}(n)}\right\} .
\end{aligned}
$$

Also, one has

$$
\begin{aligned}
& V_{1}(n+1) \leq\left(1-\eta_{1}^{l}\right) V_{1}(n)+q_{1}^{u}\left|x_{1}(n)-x_{1}^{*}(n)\right|, \\
& V_{2}(n+1) \leq\left(1-\eta_{2}^{l}\right) V_{1}(n)+q_{2}^{u}\left|x_{2}(n)-x_{2}^{*}(n)\right| .
\end{aligned}
$$

We have

$$
\begin{aligned}
\left|x_{i}(n)-x_{i}^{*}(n)\right| & =\left|\exp \left(\ln x_{i}(n)\right)-\exp \left(\ln x_{i}^{*}(n)\right)\right| \\
& =\exp \left(\xi_{i}(n)\right)\left|\ln x_{i}(n)-\ln x_{i}^{*}(n)\right| \\
& =\exp \left(\xi_{i}(n)\right) W_{i}, \quad i=1,2,
\end{aligned}
$$

where $\xi_{i}(n)$ between $\ln x_{i}(n)$ and $\ln x_{i}^{*}(n)$.

As follows from the above equation, we have

$$
\begin{aligned}
W_{1}(n+1) \leq & \left|\left(\ln x_{1}(n)-\ln x_{1}^{*}(n)\right)-d_{1}(n)\left(x_{1}(n)-x_{1}^{*}(n)\right)\right|+e_{1}^{u} V_{1}(n) \\
& +\alpha_{12}^{u} \frac{\left|x_{2}(n)-x_{2}^{*}(n)\right|}{\Delta\left(n, x_{1}(n), x_{2}(n)\right)}+a^{u} \alpha_{12}^{u} x_{1}^{*}(n) \frac{\left|x_{2}(n)-x_{2}^{*}(n)\right|}{\Delta\left(n, x_{1}(n), x_{2}(n)\right)} \\
& -a(n) \alpha_{12}(n) x_{2}^{*}(n) \frac{\left|x_{1}(n)-x_{1}^{*}(n)\right|}{\Delta\left(n, x_{1}(n), x_{2}(n)\right)} \\
\leq & \left|1-d_{1}(n) \exp \left(\xi_{1}(n)\right)\right| W_{1}(n)+e_{1}^{u} V_{1}(n) \\
& +\alpha_{12}^{u} \frac{\exp \left(\xi_{2}(n)\right) W_{2}(n)}{\Delta\left(n, x_{1}(n), x_{2}(n)\right)}+a^{u} \alpha_{12}^{u} x_{1}^{*}(n) \frac{\exp \left(\xi_{2}(n)\right) W_{2}(n)}{\Delta\left(n, x_{1}(n), x_{2}(n)\right)} \\
& -a(n) \alpha_{12}(n) x_{2}^{*}(n) \frac{\exp \left(\xi_{1}(n)\right) W_{1}(n)}{\Delta\left(n, x_{1}(n), x_{2}(n)\right)}, \\
W_{2}(n+1) \leq & W_{2}(n)+b^{u} \alpha_{21}^{u} x_{2}^{*}(n) \frac{\exp \left(\xi_{1}(n)\right) W_{1}(n)}{\Delta\left(n, x_{1}(n), x_{2}(n)\right)}+e_{2}^{u} V_{2}(n) \\
& +\alpha_{21}^{u} \frac{\exp \left(\xi_{1}(n)\right) W_{1}(n)}{\Delta\left(n, x_{1}(n), x_{2}(n)\right)}-b(n) \alpha_{21}(n) x_{1}^{*}(n) \frac{\exp \left(\xi_{2}(n)\right) W_{2}(n)}{\Delta\left(n, x_{1}(n), x_{2}(n)\right)}, \\
V_{1}(n+1) \leq & \left(1-\eta_{1}^{l}\right) V_{1}(n)+q_{1}^{u} \exp \left(\xi_{1}(n)\right) W_{1}, \\
V_{2}(n+1) \leq & \left(1-\eta_{2}^{l}\right) V_{2}(n)+q_{2}^{u} \exp \left(\xi_{2}(n)\right) W_{2} .
\end{aligned}
$$


By (3.1), we can choose a $\varepsilon>0$ such that

$$
\left\{\begin{aligned}
\chi_{1}^{\varepsilon}= & \max \left\{\left|1-d_{1}^{l} m_{1 \varepsilon}-\frac{a^{l} \alpha_{12}^{l} m_{1 \varepsilon} m_{2 \varepsilon}}{\Delta\left(n, M_{\varepsilon}, M_{2 \varepsilon}\right)}\right|,\left|1-d_{1}^{u} M_{1 \varepsilon}-\frac{a^{u} \alpha_{12}^{u} M_{1 \varepsilon} M_{2 \varepsilon}}{\Delta\left(n, m_{1 \varepsilon}, M_{2 \varepsilon}\right)}\right|\right\} \\
& +\alpha_{12}^{u}\left[\frac{M_{2 \varepsilon}}{\Delta\left(n, m_{1 \varepsilon}, m_{2 \varepsilon}\right)}+\frac{a^{u} M_{1 \varepsilon} M_{2 \varepsilon}}{\Delta\left(n, M_{1 \varepsilon}, m_{2 \varepsilon}\right)}\right]+e_{1}^{u}<1, \\
\chi_{2}^{\varepsilon}= & \left.\max \left\{\mid 1-\frac{b^{l} \alpha_{21}^{l} m_{1 \varepsilon} m_{2 \varepsilon}}{\Delta\left(n, m_{1 \varepsilon}, M_{2 \varepsilon}\right.}\right),\left|1-\frac{b^{u} \alpha_{21}^{u} M_{1 \varepsilon} M_{2 \varepsilon}}{\Delta\left(n, M_{1 \varepsilon}, m_{2 \varepsilon}\right)}\right|\right\} \\
& +\alpha_{21}^{u}\left[\frac{M_{1 \varepsilon}}{\Delta\left(n, m_{1 \varepsilon}, m_{2 \varepsilon}\right)}+\frac{b^{u} M_{1 \varepsilon} M_{2 \varepsilon}}{\Delta\left(n, m_{1 \varepsilon}, M_{2 \varepsilon}\right)}\right]+e_{2}^{u}<1, \\
\chi_{3}^{\varepsilon}= & 1-\eta_{1}^{l}+q_{1}^{u} M_{1 \varepsilon}<1 \\
\chi_{4}^{\varepsilon}= & 1-\eta_{2}^{l}+q_{2}^{u} M_{2 \varepsilon}<1 .
\end{aligned}\right.
$$

In view of Theorem 2.7, there exists $N_{4}>N_{3}$ such that

$$
\begin{aligned}
& m_{i \varepsilon} \leq x_{i}(n), \quad x_{i}^{*}(n) \leq M_{i \varepsilon}, \\
& w_{i \varepsilon} \leq u_{i}(n), \quad u_{i}^{*}(n) \leq W_{i \varepsilon}, \\
& m_{i \varepsilon} \leq \exp \left(\xi_{i}(n)\right) \leq M_{i \varepsilon}, \quad i=1,2 .
\end{aligned}
$$

It follows from (3.6) that

$$
\begin{aligned}
W_{1}(n+1) \leq & \max \left\{\left|1-d_{1}^{l} m_{1 \varepsilon}-\frac{a^{l} \alpha_{12}^{l} m_{1 \varepsilon} m_{2 \varepsilon}}{\Delta\left(n, M_{1 \varepsilon}, m_{2 \varepsilon}\right)}\right|,\left|1-d_{1}^{u} M_{1 \varepsilon}-\frac{a^{u} \alpha_{12}^{u} M_{1 \varepsilon} M_{2 \varepsilon}}{\Delta\left(n, m_{1 \varepsilon}, M_{2 \varepsilon}\right)}\right|\right\} \\
& \times W_{1}(n)+\alpha_{12}^{u}\left[\frac{M_{2 \varepsilon}}{\Delta\left(n, m_{1 \varepsilon}, m_{2 \varepsilon}\right)}+\frac{a^{u} M_{1 \varepsilon} M_{2 \varepsilon}}{\Delta\left(n, M_{1 \varepsilon}, m_{2 \varepsilon}\right)}\right] W_{2}(n)+e_{1}^{u}(n) V_{1}(n), \\
W_{2}(n+1) \leq & \max \left\{\left|1-\frac{b^{l} \alpha_{21}^{l} m_{1 \varepsilon} m_{2 \varepsilon}}{\Delta\left(n, m_{1 \varepsilon}, M_{2 \varepsilon}\right)}\right|,\left|1-\frac{b^{u} \alpha_{21}^{u} M_{1 \varepsilon} M_{2 \varepsilon}}{\Delta\left(n, M_{1 \varepsilon}, m_{2 \varepsilon}\right)}\right|\right\} W_{2}(n) \\
& +\alpha_{21}^{u}\left[\frac{M_{1 \varepsilon}}{\Delta\left(n, m_{1 \varepsilon}, m_{2 \varepsilon}\right)}+\frac{b^{u} M_{1 \varepsilon} M_{2 \varepsilon}}{\Delta\left(n, m_{1 \varepsilon}, M_{2 \varepsilon}\right)}\right] W_{1}(n)+e_{2}^{u}(n) V_{2}(n) \\
V_{1}(n+1) \leq & \left(1-\eta_{1}^{l}(n)\right) V_{1}(n)+q_{1}^{u}(n)(M+\varepsilon) W_{1}(n) \\
V_{2}(n+1) \leq & \left(1-\eta_{2}^{l}(n)\right) V_{2}(n)+q_{2}^{u}(n)(M+\varepsilon) W_{2}(n) .
\end{aligned}
$$

Let $\chi=\max \left\{\chi_{1}^{\varepsilon}, \chi_{2}^{\varepsilon}, \chi_{3}^{\varepsilon}, \chi_{4}^{\varepsilon}\right\}$, then $0<\chi<1$. It follows from (3.7) that

$$
\begin{aligned}
& \max \left\{W_{1}(n+1), W_{2}(n+1), V_{1}(n+1), V_{2}(n+1)\right\} \\
& \quad \leq \chi \max \left\{W_{1}(n), W_{2}(n), V_{1}(n), V_{2}(n)\right\}
\end{aligned}
$$

for $n>N_{4}$. Then we have

$$
\begin{gathered}
\max \left\{W_{1}(n+1), W_{2}(n+1), V_{1}(n+1), V_{2}(n+1)\right\} \\
\leq \chi^{n-N_{4}} \max \left\{W_{1}(n), W_{2}(n), V_{1}(n), V_{2}(n)\right\} .
\end{gathered}
$$

Thus

$$
\lim _{n \rightarrow+\infty} W_{i}(n)=0, \quad \lim _{n \rightarrow+\infty} V_{i}(n)=0, \quad i=1,2 .
$$

This completes the proof. 


\section{Examples}

In the section, we present some examples showing the feasibility of our main results.

Example 4.1 Consider the following system:

$$
\begin{aligned}
& x_{1}(n+1)=x_{1}(n) \exp \left\{1-0.2 x_{1}(n)+\frac{(0.9+0.1 \cos (n)) x_{2}(n)}{1+x_{1}(n)+x_{2}(n)}-0.1 u_{1}(n)\right\} \\
& x_{2}(n+1)=x_{2}(n) \exp \left\{-0.5+\frac{(1.45+0.05 \sin (n)) x_{1}(n)}{1+x_{1}(n)+x_{2}(n)}-0.2 u_{2}(n)\right\} \\
& \Delta u_{1}(n)=-0.8 u_{1}(n)+0.2 x_{1}(n) \\
& \Delta u_{2}(n)=-0.5 u_{2}(n)+0.1 x_{2}(n) .
\end{aligned}
$$

Through a simple computation, we have

$$
-r_{2}^{l}+\frac{\alpha_{21}^{u}}{a^{l}}=1>0 .
$$

So Proposition 2.6 holds, moreover,

$$
-r_{2}^{u}+\frac{\alpha_{21}^{l} m_{1}}{1+a^{u} m_{1}}-e_{1}^{u} W_{2} \approx 0.0474>0 .
$$

Therefore the conditions of Theorem 2.7 hold. Then the system (4.1) has permanence.

Numeric simulation (Figure 1) also supports this finding.

Example 4.2 Consider the following system:

$$
\begin{aligned}
x_{1}(n+1)= & x_{1}(n) \exp \left\{0.15+0.05 \cos (n)-2.5 x_{1}(n)\right. \\
& \left.+\frac{(0.6+0.1 \cos (n)) x_{2}(n)}{1+x_{1}(n)+2 x_{2}(n)}-0.6 \cos (n) u_{1}(n)\right\},
\end{aligned}
$$

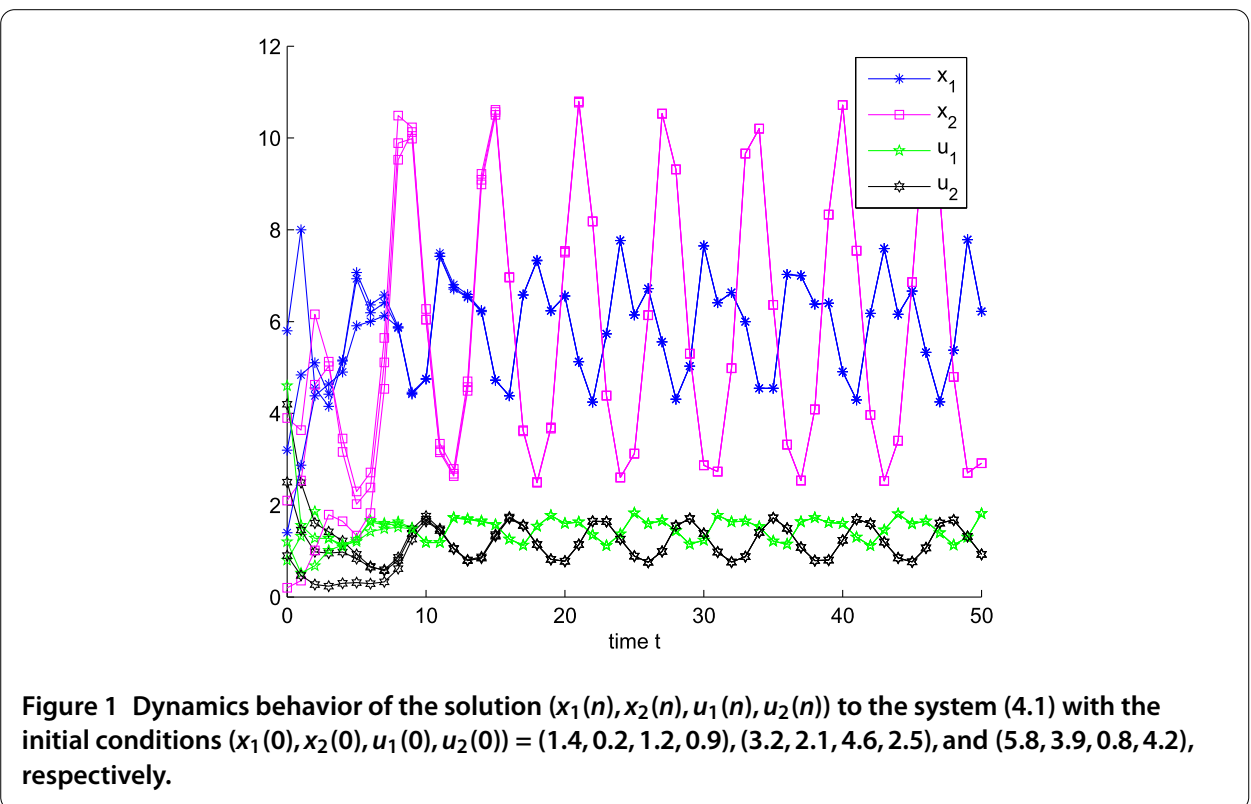




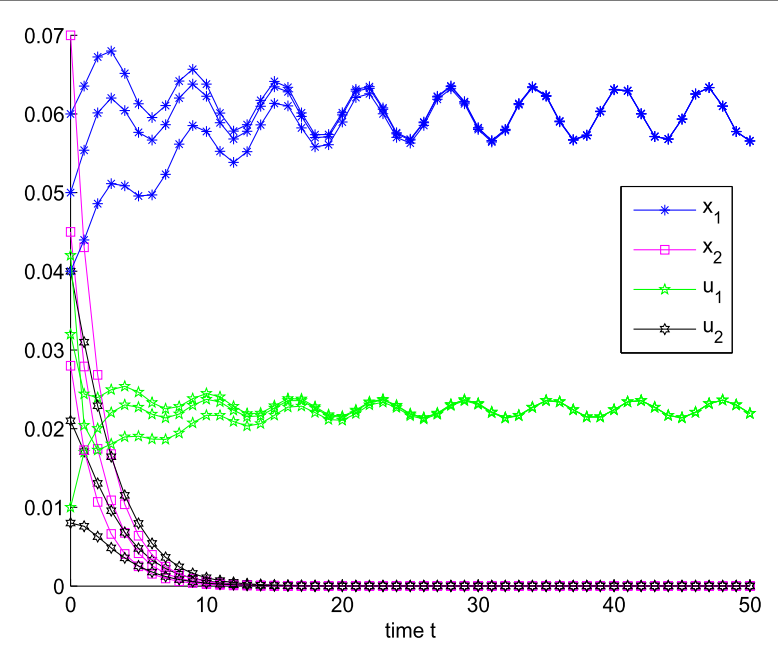

Figure 2 Dynamics behavior of the solution $\left(x_{1}(n), x_{2}(n), u_{1}(n), u_{2}(n)\right)$ of the system (4.2) with the initial conditions $\left(x_{1}(0), x_{2}(0), u_{1}(0), u_{2}(0)\right)=(0.05,0.07,0.01,0.04),(0.06,0.045,0.032,0.021)$, and $(0.04,0.028,0.042,0.008)$, respectively.

$$
\begin{aligned}
& x_{2}(n+1)=x_{2}(n) \exp \left\{-0.5+\frac{0.1 \sin (n) x_{1}(n)}{1+x_{1}(n)+2 x_{2}(n)}-0.3 \cos (n) u_{2}(n)\right\}, \\
& \Delta u_{1}(n)=-0.8 u_{1}(n)+0.3 x_{1}(n) \\
& \Delta u_{2}(n)=-0.4 u_{2}(n)+0.1 x_{2}(n) .
\end{aligned}
$$

Through a simple computation, we have

$$
\begin{aligned}
& r_{2}^{l}=0.5>0.1=\frac{\alpha_{21}^{u}}{a^{l}}, \\
& \eta_{1}^{l}=0.8>0.6=e_{1}^{u}, \\
& \min \left[d_{1}^{l}, \frac{2}{M_{1}}-d_{1}^{u}\right]=2.5>0.3=q_{1}^{u} .
\end{aligned}
$$

Therefore the conditions of Theorems 2.8 and 2.9 hold. Then the species $x_{2}$ is extinct and the species $x_{1}$ has global stability.

Numeric simulation (Figure 2) also supports this finding.

\section{Conclusion}

In this paper, we proposed a discrete non-autonomous plant-pollinator system with the Beddington-DeAngelis functional response and feedback controls. As we see it, plants can build a cooperative interaction with pollinators by providing a reward for the pollinators' services. From Theorem 2.7, we discover that when $\alpha_{21}(n)$ is large enough, then (2.10) must hold, that is, the system (1.4) has an upper bound. We know that $\alpha_{21}(n)$ represents the pollinators' efficiency in translating plant-pollinator interactions into fitness. In other words, when the efficiency of the pollinators is large enough, the system has an upper bound. What is more, when the coefficient $e_{1}(n)$ is small enough, then the system has permanence. That is, when the interference of the plant is small, the system is to persist. From Theorem 2.8, it is obvious that when $r_{2}(n)$ is large enough, $x_{2}(n)$ will contribute 
to extinction. That is, if the mortality is large enough, then the population will go to extinction. From Theorem 2.9, we known that when $e_{1}(n)$ is small enough, then the partial species is globally stable. That is, if the feedback control is small enough, the population may remain stable. Wang et al. [10] have shown that the system (1.3) is globally stable, and our work shows that the feedback controls have no influence on the attractivity of the system. The obtained results may be helpful to maintain the plant-pollinator cooperation and provide insight in the mechanisms by which pollination mutualism could persist and we have global attractivity, which may be helpful for understanding the complexity of these systems.

\section{Competing interests}

The authors declare that they have no competing interests.

\section{Authors' contributions}

All authors contributed to the writing of this paper. All authors read and approved the final manuscript.

\section{Author details}

'College of Mathematics and Computer Science, Fuzhou University, Fuzhou, Fujian 350116, P.R. China. ${ }^{2}$ Department of Mathematics, Ningde Normal University, Ningde, Fujian 352300, P.R. China.

\section{Acknowledgements}

The authors are grateful to the anonymous referees for their excellent suggestions, which greatly improved the presentation of the paper. The research was supported by the Natural Science Foundation of Fujian Province (2015J010121, 2015J01019)

Received: 30 August 2015 Accepted: 6 June 2016 Published online: 28 July 2016

\section{References}

1. Beddington, JR: Mutual interference between parasites or predator and its effect on searching efficiency. J. Anim. Ecol. 44(1), 331-340 (1975)

2. DeAngelis, DL, Goldstein, RA, O’Neil, RV: A model for tropic interaction. Ecology 56(4), 881-892 (1975)

3. Chen, FD, You, MS: Permanence, extinction and periodic solution of the predator-prey system with Beddington-DeAngelis functional response and stage structure for prey. Nonlinear Anal., Real World Appl. 9(2), 207-221 (2008)

4. Xiao, HB: Positive equilibrium and its stability of the Beddington-DeAngelis' type predator-prey dynamical system. Appl. Math. J. Chin. Univ. Ser. B 21(4), 429-436 (2006)

5. Khajanchi, S: Dynamic behavior of a Beddington-DeAngelis type stage structured predator-prey model. Appl. Math. Comput. 244, 344-360 (2014)

6. Chen, FD, Chen, YM, Shi, JL: Stability of the boundary solution of a nonautonomous predator-prey system with the Beddington-DeAngelis functional response. J. Math. Anal. Appl. 344(2), 1057-1067 (2008)

7. $\mathrm{Wu}, \mathrm{T}$ : Permanence and global stability of a discrete competition feedback-control system with Beddington-DeAngelis functional response. J. Minjiang Univ. 31(2), 16-20 (2010) (in Chinese)

8. Wu, T: Permanence of a discrete $n$-species competition-predator system with Beddington-DeAngelis functional response. Pure Appl. Math. 27(4), 437-441 (2011) (in Chinese)

9. Li, XP, Yang, WS: Permanence of a discrete predator-prey systems with Beddington-DeAngelis functional response and feedback control. Discrete Dyn. Nat. Soc. 2008, Article ID 149267 (2008)

10. Wang, YS, Wu, H, Sun, S: Persistence of pollination mutualisms in plant-pollination-robber systems. Theor. Popul. Biol. 81, 243-250 (2012)

11. Huo, HF, Li, WT: Positive periodic solution of a class of delay differential system with feedback control. Appl. Math. Comput. 148(1), 35-46 (2004)

12. Chen, FD, Xie, XD: Study on the Dynamic Behaviors of Cooperation Population Modeling. Science Press, Beijing (2014) (in Chinese)

13. Takeuchi, Y: Global Dynamical Properties of Lotka-Volterra Systems. World Scientific, Singapore (1996)

14. Fan, YH, Wang, LL: Permanence for a discrete model with feedback control and delay. Discrete Dyn. Nat. Soc. 2008, Article ID 945109 (2008)

15. Chen, LJ, Chen, FD: Extinction in a discrete Lotka-Volterra competitive system with the effect of toxic substances and feedback controls. Int. J. Biomath. 8(1), 1550012 (2015) 